\title{
Intrinsic nonlinear effects of dipole magnets in small rings
}

\author{
H. S. Xu, ${ }^{1, *}$ W. H. Huang, ${ }^{1}$ C. X. Tang, ${ }^{1}$ and S. Y. Lee ${ }^{2}$ \\ ${ }^{1}$ Accelerator Laboratory, Department of Engineering Physics, Tsinghua University, \\ Beijing 100084, China \\ ${ }^{2}$ Department of Physics, Indiana University, Bloomington, Indiana 47405, USA
}

(Received 22 February 2016; published 3 June 2016)

\begin{abstract}
We find that dynamic aperture depends significantly on the bending radii of dipole magnets when designing a small storage ring for Tsinghua Thomson scattering X-ray source (TTX) mainly because of the nonlinearity of the dipole field. In this paper, we present systematic studies on the intrinsic-geometric nonlinearity of dipole magnets. The Hamiltonian approach is used to determine the expressions of the geometric nonlinear potential and the corresponding third-order resonance strengths. Simulations are conducted to study these resonances. Our analysis results agree well with the tracking results at the thirdorder resonances $3 \nu_{x}=\ell$ and $\nu_{x} \pm 2 \nu_{z}=\ell$, where $\ell$ 's are the integer multiple of the number of superperiods.
\end{abstract}

DOI: 10.1103/PhysRevAccelBeams.19.064001

\section{INTRODUCTION}

Compact rings, which have many applications in various research fields, such as compact light sources [1-3], boosters [4], and hadron therapy [5-8], are of great interest to many researchers mainly because of their inexpensive, flexible, and high-performance properties. They are therefore considered excellent supplements to large-scale accelerator complexes.

The idea of using a compact laser-electron storage ring in an inverse Compton scattering (ICS) X-ray source was proposed by Huang and Ruth [1]. Intense ICS X-rays can be produced at a MHz-repetition rate by the interactions between electron beams stored in a compact ring and high power laser pulses accumulated in an optical cavity. We plan to add a small electron storage ring to the existing Tsinghua Thomson scattering X-ray source (TTX) [9]. The design of a 4.8-m ring for TTX is presented in [10].

Our lattice design ideas use dipole magnets with carefully adjusted bending radii and edge angles to provide proper focusing in both transverse directions and quadrupole magnets to adjust the damping partition number and momentum compaction factor. The bending radii of the dipole magnets in the ring need to be very small because the horizontal focusing strength induced by the dipole field is inversely proportional to the square of the bending radius (this dependence is shown in Sec. II A). Meanwhile, the

\footnotetext{
*haishengxu@gmail.com

Present address: Paul Scherrer Institut, CH-5232 Villigen PSI, Switzerland.

Published by the American Physical Society under the terms of the Creative Commons Attribution 3.0 License. Further distribution of this work must maintain attribution to the author $(s)$ and the published article's title, journal citation, and DOI.
}

edge angles of the dipole magnets should be large enough to provide a strong enough vertical focusing.

At the beginning, we designed several lattices with circumferences of $2.8 \mathrm{~m}, 3.0 \mathrm{~m}, 3.6 \mathrm{~m}, 4.8 \mathrm{~m}$, and $6.0 \mathrm{~m}$, with bending radii of $0.1082 \mathrm{~m}, 0.1401 \mathrm{~m}$, $0.1464 \mathrm{~m}, 0.2546 \mathrm{~m}$, and $0.3501 \mathrm{~m}$, respectively. In these cases, the nonzero edge angles of the dipole magnets were used to adjust the vertical betatron tunes. Only the linear effects of the dipole fringe fields were considered by applying Karl L. Brown's formalism [11]. The fringe fields of the quadrupole magnets were not included in the studies. However, we found that enlarging the dynamic aperture became more and more difficult when the bending radii of dipole magnets became smaller. We started to look at the nonlinearity of the dipole field, which was eventually demonstrated the key limitation of the dynamic aperture in the lattices mentioned above because nonlinear multipole field did not exist in the lattice. We call this nonlinearity the intrinsic-geometric nonlinearity of dipole field.

Furthermore, many studies [11-16] indicated that the fringe fields of dipole magnets can influence the beam dynamics in a ring, especially when the bending radii of dipole magnets are small. In our studies [10], we have considered the linear effects of dipole fringe fields, in which the vertical gap of the dipole magnets $(\mathrm{g})$ and the fringe field integral (FINT) are variables. A Lie map generator of the dipole fringe field was derived by Hwang and Lee recently [16]. Their results show that the nonlinear effects of dipole fringe fields are critical in the TTX ring. Therefore, the serious consideration of the fringe field effects is necessary in both the optimization of lattice and the design of magnets. However, the nonlinear fringe field effects are out of the scope of our paper.

In this paper, we present our studies of the intrinsicgeometric nonlinearity of dipole magnets. We exclude all 
the other nonlinear sources, such as the nonlinearity of the dipole fringe fields and the nonlinear multipole fields, to simplify our study. The rest of this paper is organized as follows. In Sec. II, the nonlinear Hamiltonian dynamics in the Frenet-Serret coordinate system is discussed. The expressions of the new Hamiltonian and the corresponding resonance strengths in the action-angle variables are provided in this section. In Sec. III, we present the calculations of the resonance strengths using the lattices based on the TTX ring layout as examples. The resonance strengths of the third-order resonance $3 \nu_{x}=4$ and the third-order coupling resonance $\nu_{x}-2 \nu_{z}=-2$ driven by the dipole fields are calculated both analytically and by fitting the tracking data. The results from these two methods are compared. The dependence of the resonance strengths on the bending radii of dipole magnets is also studied. The conclusion is in Sec. IV.

\section{THEORY}

\section{A. Hamiltonian for particle motion}

The Hamiltonian for particle motion in the Frenet-Serret coordinate system can be expressed by (e.g., [17])

$$
\begin{aligned}
\tilde{H}= & -\left(1+\frac{x}{\rho}\right)\left[\frac{(H-e \phi)^{2}}{c^{2}}-m^{2} c^{2}-\left(p_{x}-e A_{x}\right)^{2}\right. \\
& \left.-\left(p_{z}-e A_{z}\right)^{2}\right]^{1 / 2}-e A_{s},
\end{aligned}
$$

where the six dimensional phase-space coordinates are $\left(x, p_{x}, z, p_{z}, t,-H\right), \rho$ denotes the bending radius, and $\phi$ and $\vec{A}\left(A_{x}, A_{z}, A_{s}\right)$ are the scalar potential and vector potential in the Frenet-Serret coordinate system, respectively.

Since the transverse conjugate momenta $p_{x}$ and $p_{z}$ are much smaller than the total momentum $p=\sqrt{\frac{(H-e \phi)^{2}}{c^{2}}-m^{2} c^{2}}$, we expand the Hamiltonian $\tilde{H}$ up to the second order in $p_{x}$ and $p_{z}$. The expanded Hamiltonian is as follows:

$\tilde{H} \approx-p\left(1+\frac{x}{\rho}\right)+\frac{1+x / \rho}{2 p}\left[\left(p_{x}-e A_{x}\right)^{2}+\left(p_{z}-e A_{z}\right)^{2}\right]-e A_{s}$.

As mentioned above, we need to exclude all the nonlinear multipole components (e.g., sextupole terms) to study the intrinsic-geometric nonlinearity of dipole field. Therefore, the vector potential for a ring consisting of ideal dipole and quadrupole fields can be represented by $A_{x}=A_{z}=0$ and

$$
A_{s}(x, z)=B_{0} x+\frac{1}{2 \rho} B_{0} x^{2}+\frac{1}{2} B_{1} x^{2}-\frac{1}{2} B_{1} z^{2}
$$

where $B_{0}(s)=-p /(e \rho)$ and $B_{1}(s)=\partial B_{z} / \partial x$ are the dipole field strength and quadrupole gradient, respectively.

By substituting the expressions of the vector potential $\vec{A}\left(A_{x}, A_{z}, A_{s}\right)$ mentioned above into Eq. (2) and using $\tilde{p_{x}}=$ $p_{x} / p$ and $\tilde{p}_{z}=p_{z} / p$ as the conjugate momenta, we can obtain new Hamiltonian as follows:

$$
\begin{aligned}
H & =\frac{\tilde{H}}{p}=H_{0}+V_{\text {nonlinear }} \\
H_{0} & =\left[\frac{1}{2} \tilde{p}_{x}^{2}+\frac{1}{2} K_{x}(s) x^{2}+\frac{1}{2} \tilde{p}_{z}^{2}+\frac{1}{2} K_{z}(s) z^{2}\right] \\
V_{\text {nonlinear }} & =\frac{1}{2} \frac{x}{\rho}\left(\tilde{p}_{x}^{2}+\tilde{p}_{z}^{2}\right)
\end{aligned}
$$

where $K_{x}(s)=\left(\frac{1}{\rho^{2}}-\frac{B_{1}}{B \rho}\right)$ and $K_{z}(s)=\frac{B_{1}}{B \rho}$ are the horizontal and vertical focusing functions with the dimension $\left[\mathrm{m}^{-2}\right]$, respectively, where $H_{0}$ is the unperturbed Hamiltonian for the linear betatron motion and $V_{\text {nonlinear }}$ is the nonlinear perturbing potential. Unlike the nonlinearities of multipole fields discussed in many books (e.g., Chapter 2.VII in [17]; Chapter 13 and 14 in [18]), the $V_{\text {nonlinear }}$ shown in Eq. (3) is induced by the dipole field. This term is usually negligible in the analysis of nonlinearity in a synchrotron with large $\rho$. However, the expression of $V_{\text {nonlinear }}$ shows that its importance in compact rings increases with decreased $\rho$.

\section{B. Floquet transformation}

We carry out the Floquet transformation [17] to the Hamiltonian shown in Eq. (3) to obtain a clearer picture of the particle motion. The original position-momentum variables $\left(x, \tilde{p}_{x}, z, \tilde{p}_{z}\right)$ are replaced by the action-angle variables $\left(J_{x}, \phi_{x}, J_{z}, \phi_{z}\right)$ using

$$
\begin{aligned}
x & =\sqrt{2 \beta_{x} J_{x}} \cos \left(\Phi_{x}\right) \\
\beta_{x} \tilde{p}_{x}+\alpha_{x} x & =-\sqrt{2 \beta_{x} J_{x}} \sin \left(\Phi_{x}\right), \\
z & =\sqrt{2 \beta_{z} J_{z}} \cos \left(\Phi_{z}\right) \\
\beta_{z} \tilde{p}_{z}+\alpha_{z} z & =-\sqrt{2 \beta_{z} J_{z}} \sin \left(\Phi_{z}\right),
\end{aligned}
$$

where $\Phi_{x}=\phi_{x}+\chi_{x}(s)-\nu_{x} \theta$ and $\Phi_{z}=\phi_{z}+\chi_{z}(s)-\nu_{z} \theta$ are the phase functions, $\alpha_{x}, \beta_{x}, \alpha_{z}$ and $\beta_{z}$ are the CourantSnyder parameters, $\chi_{x}(s)=\int_{0}^{s} \frac{1}{\beta_{x}} d s$ and $\chi_{z}(s)=\int_{0}^{s} \frac{1}{\beta_{z}} d s$ are the betatron phases, $\nu_{x}$ and $\nu_{z}$ are betatron tunes, and $\theta=s / R$ denotes the orbiting angle around the ring, while $R$ is the mean radius of the ring.

The resulting Hamiltonian in action-angle variables can be written as $\bar{H}=\bar{H}_{0}+\bar{V}_{\text {nonlinear }}$, where the unperturbed Hamiltonian is $\bar{H}_{0}=R H_{0}=\nu_{x} J_{x}+\nu_{z} J_{z}$, depending only on action variables $J_{x}$ and $J_{z}$. The unperturbed Hamiltonian $\bar{H}_{0}$ is a constant in the new action-angle coordinate system because $\theta$ is the new independent "time" variable after the transformation.

The Floquet transformation of the nonlinear perturbing potential is 


$$
\begin{aligned}
\bar{V}_{\text {nonlinear }}= & \frac{\sqrt{\beta_{x} J_{x}}}{\sqrt{2} \rho}\left\{\left[\frac{\alpha_{x}^{2} J_{x}}{2 \beta_{x}}-\frac{J_{x}}{2 \beta_{x}}\right] \cos 3 \Phi_{x}+\left[\frac{\alpha_{x} J_{x}}{\beta_{x}}\right] \sin 3 \Phi_{x}\right. \\
& +\left[\frac{\alpha_{x} J_{x}}{\beta_{x}}\right] \sin \Phi_{x}+\left[\frac{3 \alpha_{x}^{2} J_{x}}{2 \beta_{x}}+\frac{J_{x}}{2 \beta_{x}}+\frac{\alpha_{z}^{2} J_{z}}{\beta_{z}}+\frac{J_{z}}{\beta_{z}}\right] \cos \Phi_{x} \\
& +\left[\frac{\alpha_{z}^{2} J_{z}}{2 \beta_{z}}-\frac{J_{z}}{2 \beta_{z}}\right] \cdot \cos \left(\Phi_{x}+2 \Phi_{z}\right)+\left[\frac{\alpha_{z} J_{z}}{\beta_{z}}\right] \cdot \sin \left(\Phi_{x}+2 \Phi_{z}\right) \\
& \left.+\left[\frac{\alpha_{z}^{2} J_{z}}{2 \beta_{z}}-\frac{J_{z}}{2 \beta_{z}}\right] \cdot \cos \left(\Phi_{x}-2 \Phi_{z}\right)-\left[\frac{\alpha_{z} J_{z}}{\beta_{z}}\right] \cdot \sin \left(\Phi_{x}-2 \Phi_{z}\right)\right\} .
\end{aligned}
$$

Given that the nonlinear perturbing potential $\bar{V}_{\text {nonlinear }}$ is a periodic function of $s$, it can be expanded in Fourier harmonics as follows:

$$
\begin{aligned}
\bar{V}_{\text {nonlinear }}= & \sum_{\ell}\left\{g_{3,0,3,0, \ell} J_{x}^{3 / 2} \cos \left(3 \phi_{x}-\ell \theta+\xi_{3,0,3,0, \ell}\right)\right. \\
& +g_{1,0,3,0, \ell} J_{x}^{3 / 2} \cos \left(\phi_{x}-\ell \theta+\xi_{1,0,3,0, \ell}\right) \\
& +g_{1,0,1,2, \ell} J_{x}^{1 / 2} J_{z} \cos \left(\phi_{x}-\ell \theta+\xi_{1,0,1,2, \ell}\right) \\
& +g_{1,2,1,2, \ell} J_{x}^{1 / 2} J_{z} \cos \left(\phi_{x}+2 \phi_{z}-\ell \theta+\xi_{1,2,1,2, \ell}\right) \\
& \left.+g_{1,-2,1,2, \ell} J_{x}^{1 / 2} J_{z} \cos \left(\phi_{x}-2 \phi_{z}-\ell \theta+\xi_{1,-2,1,2, \ell}\right)\right\}
\end{aligned}
$$

where the coefficients are

$$
\begin{aligned}
g_{3,0,3,0, \ell} e^{j \xi_{3,0,3,0, \ell}} & =\frac{\sqrt{2}}{8 \pi} \oint \frac{\alpha_{x}^{2}-1-2 j \alpha_{x}}{\rho \beta_{x}^{1 / 2}} e^{j\left[3 \chi_{x}(s)-\left(3 \nu_{x}-\ell\right) \theta\right]} d s \\
g_{1,0,3,0, \ell} e^{j \xi_{1,0,3,0, \ell}} & =\frac{\sqrt{2}}{8 \pi} \oint \frac{3 \alpha_{x}^{2}+1-2 j \alpha_{x}}{\rho \beta_{x}^{1 / 2}} e^{j\left[\chi_{x}(s)-\left(\nu_{x}-\ell\right) \theta\right]} d s \\
g_{1,0,1,2, \ell} e^{j \xi_{1,0,1,2, \ell}} & =\frac{\sqrt{2}}{4 \pi} \oint \frac{\left(\alpha_{z}^{2}+1\right) \beta_{x}^{1 / 2}}{\rho \beta_{z}} e^{j\left[\chi_{x}(s)-\left(\nu_{x}-\ell\right) \theta\right]} d s \\
g_{1,2,1,2, \ell} e^{j \xi_{1,2,1,2, \ell}} & =\frac{\sqrt{2}}{8 \pi} \oint \frac{\left(\alpha_{z}^{2}-1-2 j \alpha_{z}\right) \beta_{x}^{1 / 2}}{\rho \beta_{z}} e^{j\left[x_{x}(s)+2 \chi_{z}(s)-\left(\nu_{x}+2 \nu_{z}-\ell\right) \theta\right]} d s \\
g_{1,-2,1,2, \ell} e^{j \xi_{1,-2,1,2, \ell}} & =\frac{\sqrt{2}}{8 \pi} \oint \frac{\left(\alpha_{z}^{2}-1+2 j \alpha_{z}\right) \beta_{x}^{1 / 2}}{\rho \beta_{z}} e^{j\left[\chi_{x}(s)-2 \chi_{z}(s)-\left(\nu_{x}-2 \nu_{z}-\ell\right) \theta\right]} d s .
\end{aligned}
$$

\section{Resonances}

The nonlinear perturbing potential $\bar{V}_{\text {nonlinear }}$ in Eq. (5) shows that three kinds of resonances $\left(\nu_{x}=\ell, 3 \nu_{x}=\ell\right.$, and $\nu_{x} \pm 2 \nu_{z}=\ell$, where $\ell$ is an integer) can be driven by the dipole field.

A particle's motion is widely known to be dominated by the specific resonance when the betatron tunes of the particle are close to this resonance line. Therefore, when the betatron tunes of a particle are near the resonance line $m \nu_{x}+n \nu_{z}=\ell$, the Hamiltonian can be written in the following form:

$$
\begin{aligned}
\bar{H}= & \nu_{x} J_{x}+\nu_{z} J_{z}+g_{m, n, p, q, \ell} J_{x}^{p / 2} J_{z}^{q / 2} \\
& \cdot \cos \left(m \phi_{x}+n \phi_{z}-\ell \theta+\xi_{m, n, p, q, \ell}\right),
\end{aligned}
$$

where $m, n, p, q$, and $\ell$ are all integers; $(|m|+|n|)$ denotes the order of the resonance; $p$ and $q$ satisfy $p \geq|m|$ and $q \geq|n|$, respectively; $\xi_{m, n, p, q, \ell}$ is the resonance phase; and $g_{m, n, p, q, \ell}$ is the resonance strength. The subscripts of the resonances, which can be driven by the dipole field, are listed in Table I. The expressions of the corresponding $g_{m, n, p, q, \ell}$ of these resonances are shown in Eq. (6), which can be used to estimate the resonance strengths driven by the dipole fields analytically. The resonance strengths shown in Eq. (6) are determined by the bending radius $\rho$, the Courant-Snyder parameters, and the resonance proximity numbers $\delta$ (for a particle near the resonance line $m \nu_{x}+n \nu_{z}=\ell, \delta=m \nu_{x}+n \nu_{z}-\ell$ ). We notice that $\rho$ is always in the denominators, which implies that the resonances tend to become stronger when the bending 
TABLE I. Types of resonances that can be driven by the dipole field and the corresponding subscripts.

\begin{tabular}{lcccc}
\hline \hline Resonances & $\mathrm{m}$ & $\mathrm{n}$ & $\mathrm{p}$ & $\mathrm{q}$ \\
\hline $3 \nu_{x}=\ell$ & 3 & 0 & 3 & 0 \\
$\nu_{x}=\ell$ & 1 & 0 & 3 & 0 \\
$\nu_{x}=\ell$ & 1 & 0 & 1 & 2 \\
$\nu_{x}+2 \nu_{z}=\ell$ & 1 & 2 & 1 & 2 \\
$\nu_{x}-2 \nu_{z}=\ell$ & 1 & -2 & 1 & 2 \\
\hline \hline
\end{tabular}

radius becomes smaller. If all the other terms remain the same, the resonance strengths are inversely proportional to the bending radius $\rho$.

The Hamiltonian shown in Eq. (7) is valid for describing the particle motion close to any resonance line, regardless if it is driven by dipole or sextupole fields. However, the expressions of $g_{m, n, p, q, \ell}$ are different in these two cases. Supposing $P$ superperiods are in a ring, the positions of the dipole fields satisfy the same periodic condition automatically. Therefore, the systematic resonances driven by dipole fields are always located at the $\ell=P \times$ integer. However, it is not as straightforward for the sextupole driven resonances because the arrangement of the sextupole magnets requires extra attention to ensure that the sextupole fields satisfy the same periodic condition.

\section{CALCULATIONS OF THE RESONANCE STRENGTHS}

The derivation of the Hamiltonian and the resonance strengths corresponding to the dipole-driven resonances are provided in the last section. In this section, we present the calculations of the resonance strengths by applying the analytical formulas shown in Eq. (6) and compare them with the results extracted from particle tracking. Integer and half-integer resonances should be avoided in designing a lattice because they are extremely dangerous. Therefore, the strength of the dipole-driven integer resonance is not calculated here. We study the third-order resonances $3 \nu_{x}=\ell$ and $\nu_{x} \pm 2 \nu_{z}=\ell$ induced by dipole fields carefully in this section.

The layout of the TTX electron storage ring, consisting of only four dipole and two quadrupole magnets, is used as an example here. The schematic drawing of the ring is shown in Fig. 1. In this example, each dipole magnet should provide a $90^{\circ}$ bending angle to the reference particle. In addition, the nonzero edge angles at both the entrance and exit of the dipole magnets provide vertical focus to the beam. Neither the nonlinear multipole magnets nor the nonlinear fringe fields of the dipole magnets are included in the lattice, which means that the dipole magnets are the only possible sources of nonlinearity. Two ideal quadrupole magnets are located at the centers of the two opposite straight sections. A lattice using the layout mentioned above has two superperiods. Therefore, a resonance is systematic when $\ell$ is an even integer.

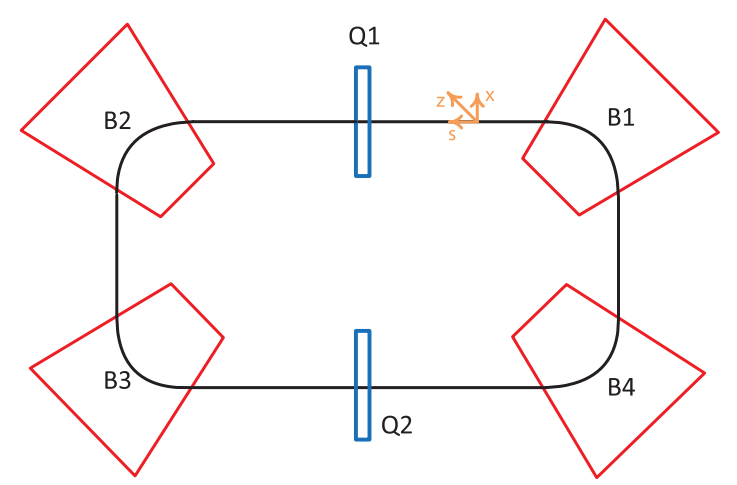

FIG. 1. Schematic of the example ring. The lattice consists of 4 dipoles (indices from B1 to B4) and 2 quadrupoles (Q1 and Q2). The Frenet-Serret coordinate system is represented by $(\mathbf{x}, \mathbf{z}, \mathbf{s})$.

Using the layout mentioned above, we are able to obtain many lattices, which satisfy the necessary condition for orbit stability $|\operatorname{Trace}(\mathbf{M})| \leq 2$ (M denotes the one-turn transfer matrix). The optical functions can be calculated easily. The resonance strengths can then be calculated by substituting the Courant-Snyder parameters, the betatron tunes, and $\rho$ into Eq. (6).

The resonance strengths can also be obtained by fitting the turn-by-turn tracking data to the Hamiltonian [see Eq. (7)] when the betatron tunes are adjusted closely to the specific resonance line. The fitting process near a $3 \nu_{x}=\ell$ resonance can be simplified to a $1 \mathrm{D}$ problem because the particle motion can be well approximated by a 1D Hamiltonian. However, the fitting near a coupling resonance is not as straightforward. The proper canonical transformation needs to be carried out first to decouple the equations of motion.

The code ELEGANT [19] is selected for the calculations of the optical functions and tracking because ELEGANT performs symplectic integration for hard-edge dipole magnets using the exact Hamiltonian [shown in Eq. (1)] [20] instead of the truncated Hamiltonian. In principle, all the nonlinear perturbing potential terms we require are included automatically in the tracking because of the usage of the exact Hamiltonian. The fourth-order symplectic integration method is applied in the particle tracking.

The bending radius, edge angles of the dipole magnets, quadrupole strength, and circumference are used to adjust the optical functions of the lattices, while maintaining two superperiods in each lattice.

\section{A. Third-order resonances $3 \nu_{x}=\boldsymbol{\ell}$}

Using the layout shown in Fig. 1, we achieve the design of a 4.8-m lattice with $\nu_{x}=1.2783$ and $\nu_{z}=1.5334$, where the length of each dipole magnet is $0.4 \mathrm{~m}$ (corresponding to $\rho=0.2546 \mathrm{~m}$ ), the edge angles at both the entrance and exit of the dipole magnets are $29^{\circ}$, the focusing strength of each quadrupole magnet is $K_{1} l_{Q}=0.4 \mathrm{~m}^{-1}$, and both short straight sections are $0.5 \mathrm{~m}$. The corresponding betatron 


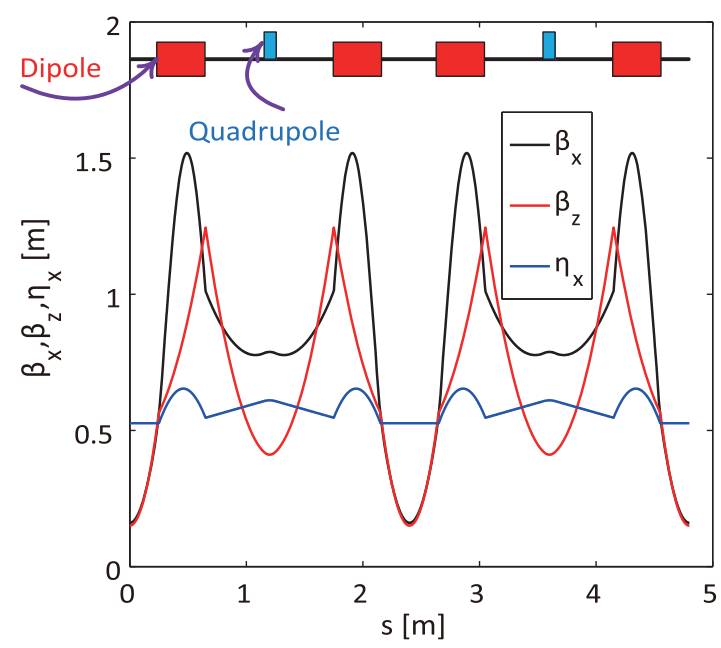

FIG. 2. Layout and the corresponding betatron amplitude and dispersion functions of a 4.8-m ring, which serves as an example to analyze the $3 \nu_{x}=\ell$ like third-order resonances.

amplitude and horizontal dispersion functions are shown in Fig. 2.

Because the horizontal bare betatron tune $\nu_{x}=1.2783$ is close to the systematic third-order resonance line $3 \nu_{x}=4$, the particle motion is dominated by this resonance. The resonance strength $g_{3,0,3,0,4}=1.0179[\pi \mathrm{m}]^{-1 / 2}$ can be obtained by substituting the values of the required optics parameters into the analytical expression of $g_{3,0,3,0, \ell}$, where $\ell$ equals 4 in this case. The calculation can be simplified by selecting the $s=0$ position properly to make sure that the resonance phase $\xi_{3,0,3,0,4}=0$.

We track several on-momentum particles with different horizontal amplitudes to calculate the resonance strength through the tracking method. The coordinates of these particles are recorded turn-by-turn. Figure 3 is the Poincaré

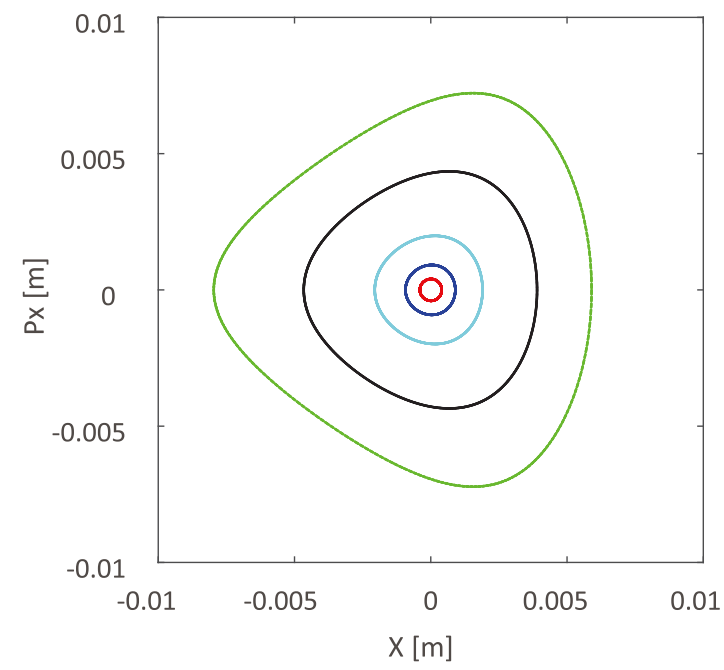

FIG. 3. Poincaré map showing the tracking data points of the particles in the horizontal normalized phase space $\left(X, P_{x}\right)$. Each color denotes the phase-space trajectory of one particle. map showing the trajectories of the tracked particles in the normalized horizontal phase space $\left(X, P_{x}\right)$, where $X=x$ and $P_{x}=\beta_{x} \tilde{p}_{x}+\alpha_{x} x$. The triangle distortion of the phasespace trajectories indicates clearly that the corresponding particles are dominated by a third-order resonance.

Since the particle motion is dominated by the resonance $3 \nu_{x}=4$, the Hamiltonian can be well-approximated by the following 1D form

$H_{3} \approx \nu_{x} J_{x}+g_{3,0,3,0,4} J_{x}^{3 / 2} \cos \left(3 \phi_{x}-\ell \theta+\xi_{3,0,3,0,4}\right)$.

After transforming the particles' coordinates $\left(x, \tilde{p}_{x}\right)$ to the action-angle variables $\left(J_{x}, \phi_{x}\right)$, we can fit the tracking data using Eq. (8) to extract the resonance strength. Both the tracking data and the fitted Hamiltonian tori are shown in Fig. 4, where the solid curves denote the fitted Hamiltonian tori, and the cross marks are the tracking data points. The fitted value of the resonance strength is $g_{3,0,3,0,4}=0.8685[\pi \mathrm{m}]^{-1 / 2}$.

We developed four more cases with different bending radii similar to the case described above. In all these cases, the horizontal betatron tune is selected to be the same value $\left(\nu_{x}=1.2783\right)$, which means that the same systematic thirdorder resonance line $3 \nu_{x}=4$ dominates. The resulting resonance strengths $g_{3,0,3,0,4}$ calculated by both analytical and tracking methods are listed in Table II. The relative differences between the fitted and analytical values of the resonance strength $g_{3,0,3,0,4}$ are also presented in the same table. We notice that the absolute values of the relative differences are always smaller than $30 \%$ in all the examples, which means that the estimation of the resonance strength $g_{3,0,3,0,4}$ by the analytical formula is reasonably good. The discrepancy between the analytical estimations and the values extracted from the tracking results may be due to the fact that more than one resonance line influences the particle motion. Even though the resonance $3 \nu_{x}=4$ may be dominant, at least one other resonance that is not negligible exists. However, we are still able to predict roughly whether the third-order resonance lines $3 \nu_{x}=\ell$

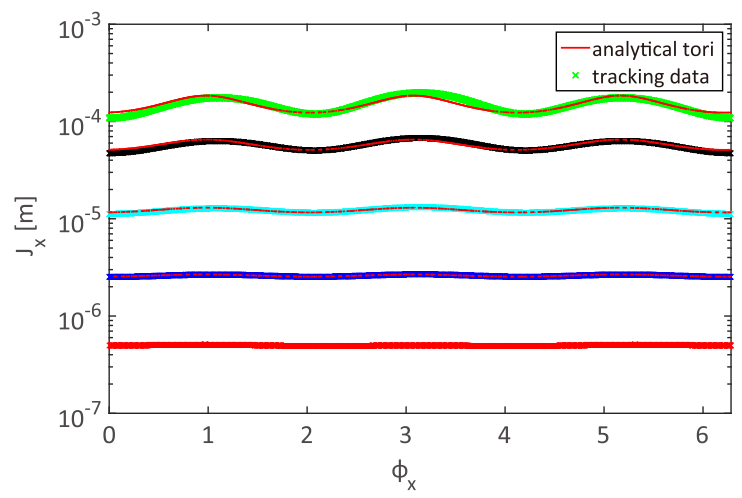

FIG. 4. Tracking data points and fitted Hamiltonian tori in the horizontal action-angle space. 
TABLE II. Comparison of the resonance strength $g_{3,0,3,0,4}$ calculated by analytical method $\left(g_{\text {ana }}\right)$ and tracking method $\left(g_{\text {fit }}\right)$ in different bending radii $\rho$.

\begin{tabular}{lcccc}
\hline \hline & $\begin{array}{c}\text { Bending } \\
\text { Radius }\end{array}$ & $\begin{array}{c}\text { Analytical } \\
\text { Resonance } \\
\text { Strength }\end{array}$ & $\begin{array}{c}\text { Fitted } \\
\text { Resonance } \\
\text { Strength }\end{array}$ & $\begin{array}{c}\text { Relative } \\
\text { Difference }\end{array}$ \\
\cline { 2 - 5 } Index & $\rho[\mathrm{m}]$ & $\begin{array}{c}g_{\text {ana }} \\
{[\pi \mathrm{m}]^{-1 / 2}}\end{array}$ & $\begin{array}{c}g_{\text {fit }} \\
{[\pi \mathrm{m}]^{-1 / 2}}\end{array}$ & $\frac{g_{\text {fit }}-g_{\text {ana }}}{g_{\text {ana }}}$ \\
\hline 1 & 0.1082 & 4.0898 & 3.4835 & $-14.82 \%$ \\
2 & 0.1401 & 2.3308 & 2.1989 & $-5.66 \%$ \\
3 & 0.1464 & 0.8642 & 0.6543 & $-24.29 \%$ \\
4 & 0.2546 & 1.0179 & 0.8685 & $-14.67 \%$ \\
5 & 0.3501 & 0.6438 & 0.8089 & $25.78 \%$ \\
\hline \hline
\end{tabular}

dominate and evaluate roughly how strong the resonances are by estimating the resonance strengths $g_{3,0,3,0, \ell}$ using the analytical formula instead of carrying out massive tracking simulations. Tracking is only required when the influences of the resonances need to be studied quantitatively.

The results in Table II show that the resonance strength is higher when the bending radius is becoming smaller, except in case 3 . This phenomena is caused by the dependence of the resonance strength not only on the bending radius but also on the Courant-Snyder parameters. We need to exclude the influences of the Courant-Snyder parameters to check the dependence of the resonance strength $g_{3,0,3,0,4}$ on the bending radii. Therefore, 100 stable lattices are randomly generated at each $\rho$ value, which means that the Courant-Snyder parameters are also randomly generated. The random variables are the strength of quadrupoles, edge angles of the dipole magnets, and the length of the straight sections. All the randomly generated lattices have the same horizontal betatron tunes to maintain a fair comparison. The resonance strength $g_{3,0,3,0,4}$ is calculated in each case by the analytical formula. The mean values and standard deviations of $g_{3,0,3,0,4}$, calculated at each bending radius, are represented by the cross marks and error bars in Fig. 5, respectively. The resonance

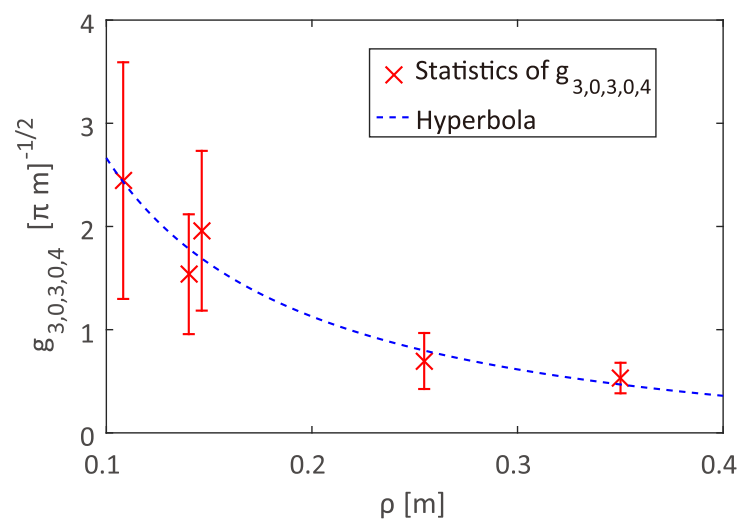

FIG. 5. Resonance strength in cases with different bending radii $\rho$. strength is inversely proportional to $\rho$ if the influences of the Courant-Snyder parameters are excluded. Therefore, we apply the hyperbolic fitting on the calculated resonance strengths. The fitted curve is represented by the blue dashed curve in Fig. 5. The good agreement between the calculated resonance strengths and the fitting curve shows that the resonance strength $g_{3,0,3,0,4}$ tends to be inversely proportional to the bending radius of the dipole magnets. The larger standard deviation of resonance strength at the smaller $\rho$ indicates that the nonlinearity becomes more sensitive to the lattice design.

\section{B. Third-order coupling resonances $\nu_{x}-2 \nu_{z}=\mathscr{C}$}

When the betatron tunes are adjusted closely to the thirdorder coupling resonance lines $\nu_{x}-2 \nu_{z}=\ell$, the coupling Hamiltonian can be expressed by

$$
\begin{aligned}
H_{3 \text { rd-Coupling }} \approx & \nu_{x} J_{x}+\nu_{z} J_{z}+g_{1,-2,1,2, \ell} J_{x}^{1 / 2} J_{z} \\
& \times \cos \left(\phi_{x}-2 \phi_{z}-\ell \theta+\xi_{1,-2,1,2, \ell}\right)
\end{aligned}
$$

which is no longer a 1D Hamiltonian. Therefore, fitting the tracking data to this Hamiltonian is very difficult. A proper canonical transformation needs to be carried out to decouple the particle motion and determine the proper way to extract the resonance strength $g_{1,-2,1,2, \ell}$.

We can carry out the canonical transformation using the second type of generating function

$$
F_{2}\left(\phi_{x}, \phi_{z}, J_{1}, J_{2}\right)=\left(\phi_{x}-2 \phi_{z}-\ell \theta+\xi\right) J_{1}+\phi_{z} J_{2},
$$

where $\phi_{x}$ and $\phi_{z}$ denote the old phase variables, corresponding to the old action variables $J_{x}$ and $J_{z}$, and $J_{1}$ and $J_{2}$ denote the new action variables corresponding to the new phase variables $\phi_{1}$ and $\phi_{2}$.

The transformation between the old and the new actionangle variables can be expressed by

$$
\begin{aligned}
J_{x} & =\frac{\partial F_{2}}{\partial \phi_{x}}=J_{1} \\
J_{z} & =\frac{\partial F_{2}}{\partial \phi_{z}}=J_{2}-2 J_{1} \\
\phi_{1} & =\frac{\partial F_{2}}{\partial J_{1}}=\left(\phi_{x}-2 \phi_{z}-\ell \theta+\xi\right) \\
\phi_{2} & =\frac{\partial F_{2}}{\partial J_{2}}=\phi_{z} .
\end{aligned}
$$

Therefore, the new Hamiltonian in the new action-angle variables is

$$
\begin{aligned}
H_{\text {new }} & =H_{3 \text { rd-Coupling }}+\frac{\partial F_{2}}{\partial \theta} \\
& =\delta J_{1}+\nu_{z} J_{2}+g_{1,-2,1,2, \ell} J_{1}^{1 / 2}\left(J_{2}-2 J_{1}\right) \cos \phi_{1}
\end{aligned}
$$




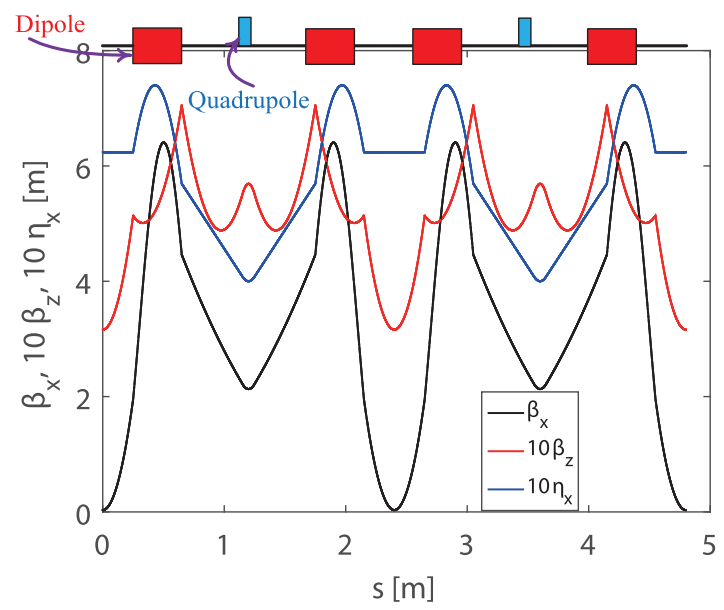

FIG. 6. Betatron amplitude and dispersion functions of a $4.8 \mathrm{~m}$ ring, which is used to study the third-order coupling resonance at $\nu_{x}-2 \nu_{z}=\ell$.
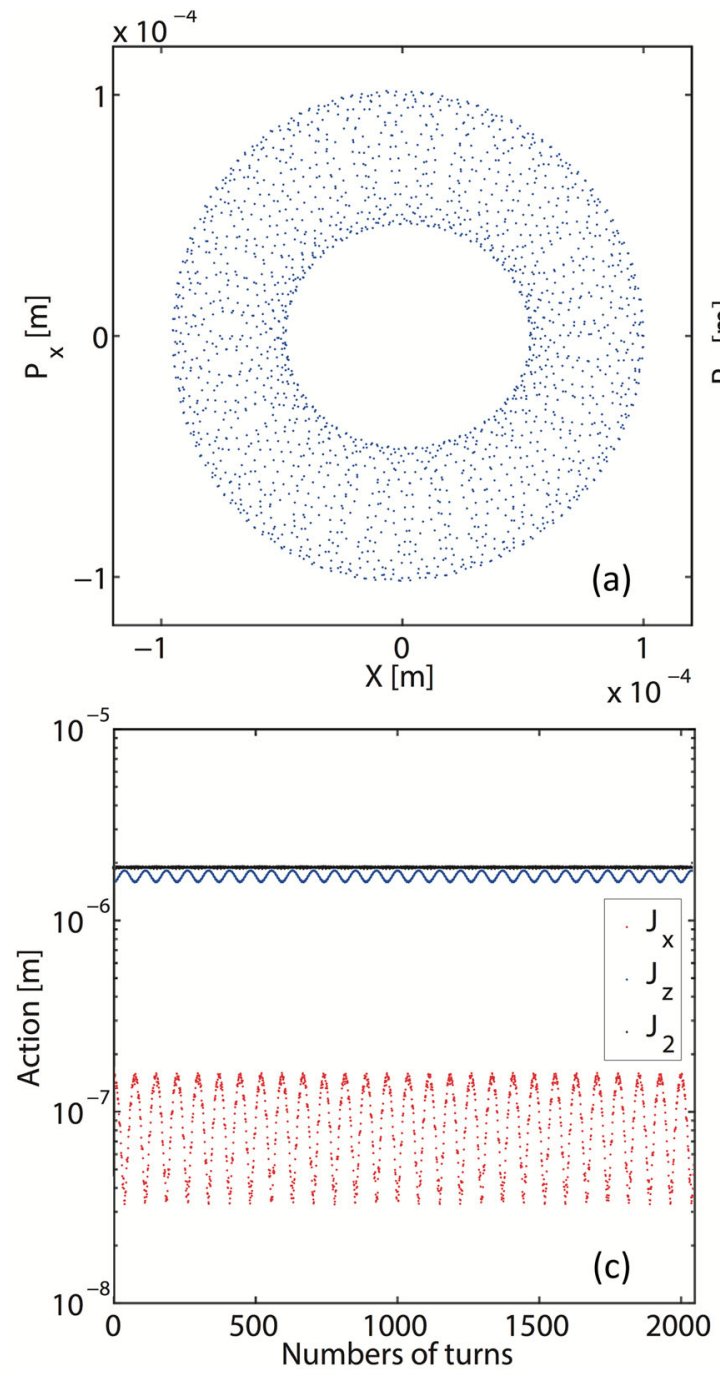

where $\delta=\nu_{x}-2 \nu_{z}-\ell$ is the resonance proximity parameter. Because the Hamiltonian $H_{\text {new }}$ is independent of $\phi_{2}$, we obtain $\dot{J}_{2}=-\partial H_{\text {new }} / \partial \phi_{2}=0$ by the Hamilton's equation, which means that $J_{2}=2 J_{x}+J_{z}$ is an invariant.

The equation of motion becomes

$\dot{J}_{1}=\frac{d J_{1}}{d \theta}=-\frac{\partial H_{\text {new }}}{\partial \phi_{1}}=g_{1,-2,1,2, \ell} J_{1}^{1 / 2}\left(J_{2}-2 J_{1}\right) \sin \phi_{1}$

which shows that the equation of motion in the new actionangle variables is not coupled.

We then design a $4.8-\mathrm{m}$ ring using the layout shown in Fig. 1 to study the coupling resonances $\nu_{x}-2 \nu_{z}=\ell$. The betatron tunes are $\nu_{x}=1.0889$ and $\nu_{z}=1.5379$ (close to the resonance $\nu_{x}-2 \nu_{z}=-2$ ) when the length of each dipole magnet is $0.4 \mathrm{~m}$ (corresponding to $\rho=0.2546 \mathrm{~m}$ ), the edge
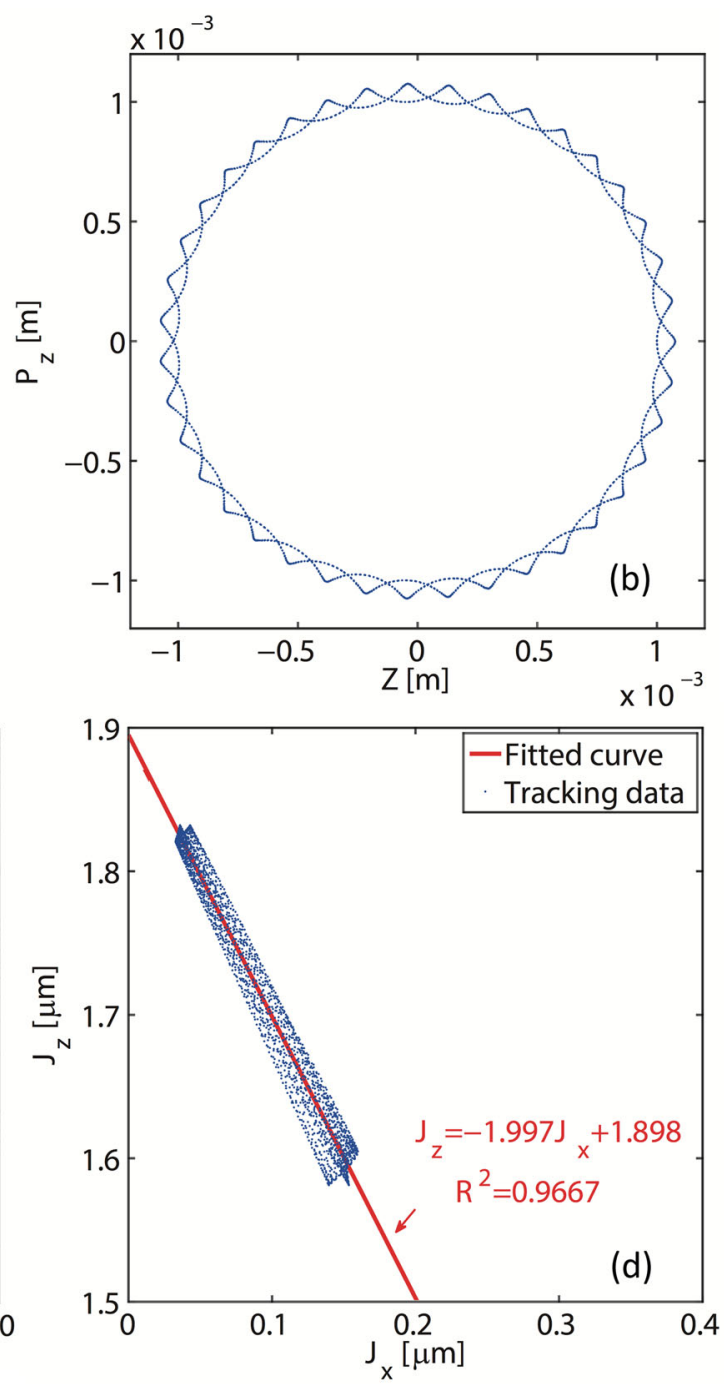

FIG. 7. The upper two plots are the horizontal and vertical normalized phase space, respectively. The lower left plot shows the actions $J_{x}, J_{z}$, and $J_{2}$ vs turns. The lower right plot shows the tracking data of the test particle, the corresponding linear fit equation, and the $\mathrm{R}$-squared of the fitting. 


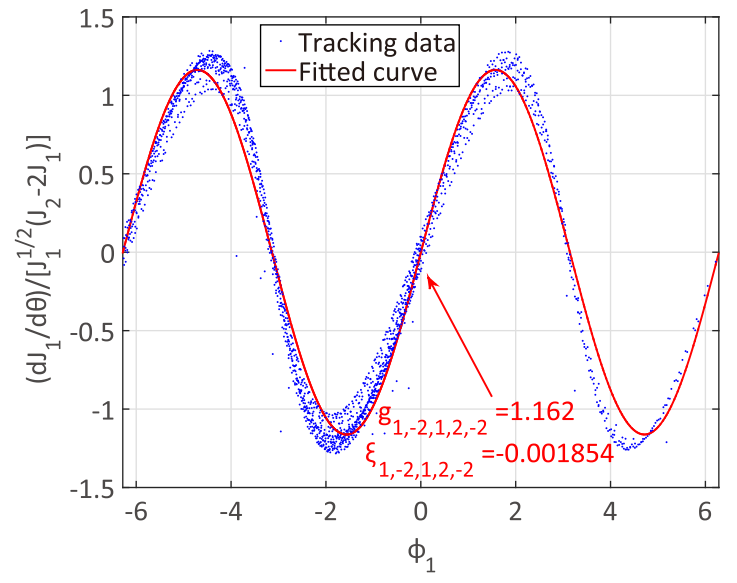

FIG. 8. Tracking data and the corresponding fitted curve.

angles at both the entrance and exit of the dipole magnets are $26.77^{\circ}$, the FINT value is 0.2 , and the focusing strength of each quadrupole magnet is $K_{1} l_{Q}=-1.6 \mathrm{~m}^{-1}$. The corresponding betatron amplitude and horizontal dispersion functions are shown in Fig. 6. By substituting the necessary optical parameters of this lattice into Eq. (6), we can obtain the resonance strength $g_{1,-2,1,2,-2}=1.166[\pi \mathrm{m}]^{-1 / 2}$.

To study this coupling resonance by tracking, we select a test particle with the following initial coordinates: $x_{\text {ini }}=0.1 \mathrm{~mm}, z_{\text {ini }}=1 \mathrm{~mm}$, and $x_{\text {ini }}^{\prime}=z_{\text {ini }}^{\prime}=0$. The nonzero initial betatron amplitudes in both the horizontal and vertical directions are necessary to study the coupled motion.

The Poincaré maps of the test particle in the horizontal and vertical normalized phase spaces are shown in Figs. 7(a) and (b). Both the horizontal and vertical phase-space trajectories are smeared because of the coupling. Figure 7(c) shows the old and new actions turn-by-turn. The $J_{x}$ and $J_{z}$ are out of phase because of the coupling. $J_{2}$ seems an invariant, which shows qualitatively that the particle motion is dominated by the resonance $\nu_{x}-2 \nu_{z}=\ell$. The turn-by-turn data points of the particle are recorded in the $\left(J_{x}, J_{z}\right)$ space (shown as the blue points in Fig. 7(d)) to calculate the value of the new invariant $J_{2}$. Furthermore, linear fitting to the tracking data is carried out, causing the equation of the fitting curve to be $J_{z}+1.997 J_{x}=1.898[\mu \mathrm{m}]$, which agrees perfectly with the theoretical prediction $J_{z}+2 J_{x}=J_{2}=$ invariant. The R-squared value of this fitting is 0.9667 , which is reasonably good.

To extract the resonance strength $g_{1,-2,1,2, \ell}$ from the tracking data, we can fit the data using Eq. (12), where $\dot{J}_{1}=d J_{1} / d \theta$ is the derivative of $J_{1}$ to the orbiting angle $\theta$, and $\phi_{1}=\phi_{x}-2 \phi_{z}+2 \theta+\xi_{1,-2,1,2, \ell}$. Because we record the particle's coordinates turn-by-turn, the derivative $\dot{J}_{1}$ can be approximated by $\left(J_{1(n+1)}-J_{1(n-1)}\right) /(2 \cdot 2 \pi)(n \geq 2)$. We can obtain the following equation by moving the term $J_{1}^{1 / 2}\left(J_{2}-2 J_{1}\right)$ to the left-hand side of the Eq. (12).

$$
\frac{\dot{J}_{1}}{J_{1}^{1 / 2}\left(J_{2}-2 J_{1}\right)}=g_{1,-2,1,2, \ell} \cdot \sin \phi_{1}
$$

which can be used directly in fitting the tracking data. Both the resonance strength $g_{1,-2,1,2, \ell}$ and phase $\xi_{1,-2,1,2, \ell}$ can be obtained [21].

The turn-by-turn data points and the fitted curve are shown in Fig. 8. The two fitted values are $g_{1,-2,1,-2,-2}=$ $1.162[\pi \mathrm{m}]^{-1 / 2}$ and $\xi_{1,-2,1,2,-2}=-0.001854$, respectively. The fitted resonance strength agrees excellently with the analytical value $g_{1,-2,1,2,-2}=1.166[\pi \mathrm{m}]^{-1 / 2}$.

\section{CONCLUSION}

In this paper, we present our study of the intrinsicgeometric nonlinearity of dipole magnets in small rings. We derive the general form of the Hamiltonian near the dipoledriven resonances in action-angle variables. The analysis shows that three kinds of resonances $\left(\nu_{x}=\ell, 3 \nu_{x}=\ell\right.$, and $\nu_{x} \pm 2 \nu_{z}=\ell$, where $\ell$ is an integer) can be driven by the dipole field. The analytical expressions of the strengths of these resonances are provided.

We use the layout of the TTX storage ring as an example to calculate the strengths of the third-order resonances $3 \nu_{x}=\ell$ and $\nu_{x} \pm 2 \nu_{z}=\ell$ both analytically and by simulation and examine the validity of the analytical results. Particle tracking is carried out by the ELEGANT code when the betatron tunes move closely to the systematic resonance lines. The third-order resonance $3 \nu_{x}=4$ and the thirdorder coupling resonance $\nu_{x}-2 \nu_{z}=-2$ are the two resonance lines we use in this paper. The calculation results show that the strengths of these two resonances extracted from tracking agree reasonably well with the values calculated analytically.

\section{ACKNOWLEDGMENTS}

This work was supported by the National Nature Science Foundation of China (Grants No. 11475097 and 11375097) and the National Key Scientific Instrument and Equipment Development Project of China (Grants No. 2013YQ1203454). One of the authors (SYL) is supported in part by grants from the U.S. Department of Energy under Contracts No. DE-FG02-12ER41800, and the National Science Foundation (NSF) No. PHY-1504778.

[1] Z. Huang and R. D. Ruth, Laser-Electron Storage Ring, Phys. Rev. Lett. 80, 976 (1998).

[2] J. Abendroth et al., X-ray structure determination of the glycine cleavage system protein $\mathrm{H}$ of Mycobacterium tuberculosis using an inverse Compton synchrotron X-ray source, J. Struct. Funct. Genomics 11, 91 (2010). 
[3] A. Loulergue et al., in Proceedings of the International Particle Accelerator Conference, Kyoto, Japan (ICR, Kyoto, 2010), p. THPE060.

[4] D. L. Friesel and S. Y. Lee, in Proceedings of the Particle Accelerator Conference, Dallas, TX, 1995 (IEEE, New York, 1995), p. 336; X. Kang, Ph.D. Thesis, Indiana University, 1998.

[5] K. Hiramoto et al., in Proceedings of the Particle Accelerator Conference, Vancouver, BC, Canada, 1997 (IEEE, New York, 1997), p. 3813.

[6] A. Morita et al., in Proceedings of the 18th Particle Accelerator Conference, New York, 1999 (IEEE, New York, 1999), p. 2528.

[7] K. Endo et al., in Proceedings of the European Particle Accelerator Conference, Vienna, 2000 (EPS, Geneva, 2000), p. 2515.

[8] Nader Al Harbi and S. Y. Lee, Design of a compact synchrotron for medical applications, Rev. Sci. Instrum. 74, 2540 (2003).

[9] C.X. Tang et al., Tsinghua Thomson scattering X-ray source, Nucl. Instrum. Methods Phys. Res., Sect. A 608, S70 (2009).

[10] H. S. Xu, W. H. Huang, C. X. Tang, and S. Y. Lee, Design of a 4.8-m ring for inverse Compton scattering X-ray source, Phys. Rev. ST Accel. Beams 17, 070101 (2014).
[11] Karl L. Brown, Report No. SLAC-75, 1982.

[12] K. Makino, B. Erdléyi, and M. Berz, in Proceedings of the Particle Accelerator Conference, Chicago, IL, 2001 (IEEE, New York, 2001), p. 451.

[13] Y. Papaphilippou, J. Wei, and R. Talman, Deflections in magnet fringe fields, Phys. Rev. E 67, 046502 (2003).

[14] Y. Cai and Y. Nosochkov, Report No. SLAC-PUB-11181, 2005.

[15] B. D. Muratori, J. K. Jones, and A. Wolski, Analytical expressions for fringe fields in multipole magnets, Phys. Rev. ST Accel. Beams 18, 064001 (2015).

[16] K. Hwang and S. Y. Lee, Dipole fringe field thin map for compact synchrotrons, Phys. Rev. ST Accel. Beams 18, 122401 (2015).

[17] S. Y. Lee, Accelerator Physics, Third ed. (World Scientific, Singapore, 2012).

[18] H. Wiedemann, Particle Accelerator Physics, Third ed. (Springer, New York, 2007).

[19] M. Borland, Advanced Photon Source Report No. LS-287, 2000.

[20] M. Borland, Report No. AOP-TN-2010-029, Rev. 2, 2010.

[21] M. Ellison et al., Experimental measurements of invariant surface near a 2D nonlinear resonance, Phys. Rev. E 50, 4051 (1994) 